\title{
NORMAS PRELIMINARES DRIS E FAIXAS DE SUFICIÊNCIA PARA GOIABEIRA 'PALUMA'
}

\author{
HENRIQUE ANTUNES DE SOUZA², DANILO EDUARDO ROZANE ${ }^{3}$, \\ DANIEL ANGELUCCI DE AMORIM ${ }^{4}$, WILLIAM NATALE ${ }^{5}$
}

RESUMO-O diagnóstico nutricional a partir da análise do tecido foliar é um instrumento eficiente para detectar desequilíbrios e auxiliar no processo de recomendação de adubação. Assim, objetivou-se avaliar a relação entre os índices DRIS e os teores foliares de nutrientes, estabelecer teores foliares adequados de nutrientes com o DRIS e validar normas DRIS para a goiabeira 'Paluma'. Avaliou-se um pomar comercial nas condições de cultivo do Estado de São Paulo, durante seis safras de produção de frutos, provenientes de ensaios de adubação orgânica e mineral, totalizando 168 amostras. A ordem dos nutrientes limitantes por falta em ordem decrescente foram: $\mathrm{Fe}>\mathrm{K}>\mathrm{Mn}>\mathrm{Ca}>\mathrm{B}>\mathrm{N}>\mathrm{P}>\mathrm{Cu}>\mathrm{S}>\mathrm{Mg}>\mathrm{Zn}$, e limitantes por excesso em ordem decrescente foram: $\mathrm{Cu}>\mathrm{Fe}>\mathrm{P}>\mathrm{Mn}=\mathrm{Mg}>\mathrm{Zn}>\mathrm{Ca}>\mathrm{B}>\mathrm{K}>\mathrm{S}>\mathrm{N}$. As faixas adequadas, provenientes dos índices DRIS, são: 18-21; 1,5-1,7; 15-17; 8-11; 1,8-2,5 e 2,5-2,9 $\left(\mathrm{g} \mathrm{kg}^{-1}\right)$ para os macronutrientes $\mathrm{N}, \mathrm{P}, \mathrm{K}$, $\mathrm{Ca}, \mathrm{Mg}$ e S, respectivamente, e 26-38; 5-57; 54-112; 53-101 e 13-126 (mg kg-1) para os micronutrientes B, $\mathrm{Cu}, \mathrm{Fe}, \mathrm{Mn}$ e $\mathrm{Zn}$, respectivamente. A produtividade das goiabeiras esteve associada ao estado nutricional. Termos para indexação: Psidium guajava; estado nutricional; diagnóstico nutricional.

\section{PRELIMINARY DRIS NORMS AND SUFFICIENCY RANGE FOR 'PALUMA' GUAVA TREE}

\begin{abstract}
The nutritional diagnosis based on the analysis of leaf tissue is an efficient tool for detecting imbalances and help the process of fertilization recommendation. Thus, it was evaluated to establish preliminary DRIS norms, and to derive critical levels and sufficiency ranges of nutrients in the leaf tissue to Paluma guava cultivar in commercial orchards growing conditions in the State of São Paulo. It was evaluated six harvests of fruit production, from organic and mineral fertilizer, totaling 168 samples. The order of limiting nutrients for failure in descending order were: $\mathrm{Fe}>\mathrm{K}>\mathrm{Mn}>\mathrm{Ca}>\mathrm{B}>\mathrm{N}>\mathrm{P}>\mathrm{Cu}>\mathrm{S}>\mathrm{Mg}>\mathrm{Zn}$, and limiting excess in descending order were: $\mathrm{Cu}>\mathrm{Fe}>\mathrm{P}>\mathrm{Mn}=\mathrm{Mg}>\mathrm{Zn}>\mathrm{Ca}>\mathrm{B}>\mathrm{K}>\mathrm{S}>\mathrm{N}$. The tracks from the appropriate DRIS indices are: $18-21,1.5$ to $1.7,15-17,8-11,1.8$ to 2.5 and 2.5 to $2.9\left(\mathrm{~g} \mathrm{~kg}^{-1}\right)$ for the macronutrients $\mathrm{N}, \mathrm{P}, \mathrm{K}, \mathrm{Ca}, \mathrm{Mg}$ and $\mathrm{S}$, respectively, and 26-38, 5-57, 54-112, 53-101 and 13-126 (mg kg-1) for the micronutrients $\mathrm{B}, \mathrm{Cu}, \mathrm{Fe}, \mathrm{Mn}$ and $\mathrm{Zn}$, respectively. The yield of guava was related to nutritional status. Index terms: Psidium guajava; nutritional state; nutritional diagnosis.
\end{abstract}

\section{INTRODUÇÃO}

Dentre várias metodologias existentes atualmente, capazes de avaliar o estado nutricional das plantas, tem-se o Sistema Integrado de Diagnose e Recomendação (DRIS), como alternativa viável, científica e capaz de inferir respostas rápidas e práticas no decorrer do desenvolvimento das lavouras, pois é considerado um método de interpretação holístico, menos afetado por alguns fatores que influenciam no teor de nutrientes nas plantas e, além disso, é capaz de ordenar os nutrientes em sua ordem de limitação e importância naquele momento (CRESTE, 2008).
No DRIS, faz-se a comparação das relações entre nutrientes com as encontradas nas folhas de plantas de alta produtividade. As relações adequadas são chamadas de normas e, as relações entre nutrientes são comparadas entre si e com as normas, determinando-se os nutrientes deficientes, adequados ou em níveis de consumo de luxo (MALAVOLTA, 2006).

O cálculo dos índices DRIS depende, inicialmente, do estabelecimento de valorespadrão ou normas de referência. Para isto, é necessário selecionar uma população de alta produtividade (população de referência), partindo-se da premissa de que existe relação significativa entre

\footnotetext{
'(Trabalho 264-12). Recebido em: 29-10-2012. Aceito para publicação em: 31-01-2013.

${ }^{2}$ Eng. Agrônomo, Pesquisador Embrapa/CNPC. E-mail: henrique.souza@embrapa.br

${ }^{3}$ Eng. Agrônomo, Professor Doutor Unesp - Campus Experimental de Registro. E-mail: danilorozane@registro.unesp.br ${ }^{4}$ Eng. Agrônomo, Pesquisador Epamig. E-mail: danielangelucci@hotmail.com

${ }^{5}$ Eng. Agrônomo, Professor Adjunto Unesp - Campus Jaboticabal. E-mail: natale@fcav.unesp.br
} 
o suprimento de nutrientes e seus teores na planta, de modo que aumentos ou decréscimos em suas concentrações proporcionam variação na produção (NACHTIGALL;DECHEN, 2010).

Na literatura, há trabalhos que contemplam o DRIS em fruteiras, e citam-se: citros (MOURÃO FILHO; AZEVEDO, 2005; MOURÃO FILHO, 2005; SANTANA et al., 2008), uva (TERRA et al., 2007); maçã (NACHTIGALL; DECHEN, 2007); banana (TEIXEIRA et al., 2007; RODRÍGUEZ et al., 2005); cupuaçu (DIAS et al., 2010); coqueiroanão (SANTOS et al., 2004); manga (POLITI et al., 2013), e para goiabeira, cita-se o trabalho de Hundal et al. (2007) na Índia; no entanto, para condições brasileiras, ou mesmo para o Estado de São Paulo, que é um dos maiores produtores de goiabas do País, não há normas desenvolvidas.

Em estudo sobre o tamanho amostral para coleta de folhas em goaibeira, Rozane et al. (2009) constataram que a dinâmica dos nutrientes, quando da cultura em sequeiro e irrigado, varia. Ainda, independentemente do tipo de adubação empregada, seja ela orgânica (SOUZA et al., 2011), seja mineral (NATALE et al., 1995; NATALE et al., 1996a), o DRIS é uma ferramenta importante, possibilitando a verificação de deficiências e excesso na cultura, auxiliando na diagnose para a tomada de decisão.

Assim, a partir de um banco de dados de amostras foliares provenientes de plantas de goiabeira cultivar Paluma, objetivou-se avaliar a relação entre os índices DRIS e os teores foliares de nutrientes, estabelecer teores foliares adequados de nutrientes e validar normas com o DRIS.

\section{MATERIAL E MÉTODOS}

O monitoramento nutricional das goiabeiras foi realizado a partir de informações de 168 amostras foliares provenientes de ensaios com adubação orgânica e mineral em um pomar comercial, conduzido por 6 safras (2006-2010), a adubação orgânica consistiu em doses de subproduto da indústria processadora de goiabas, conforme Souza et al. (2011), e a adubação mineral, em recomendações preconizadas por Natale et al. (1996), em plantas pertencentes ao mesmo talhão. As plantas eram da cultivar Paluma (a mais plantada em todo o Brasil atualmente), propagadas vegetativamente, com idade em 2006 de 4 anos (adultas), cuja irrigação era constituída por microaspersores tipo bailarina (31 litros por hora), monitorado por tensiometria (60\% da capacidade de campo), e a água, proveniente de poço semiartesiano. $\mathrm{O}$ espaçamento das goiabeiras utilizado foi de $7 \mathrm{~m}$ entre linhas e $5 \mathrm{~m}$ entre árvo- res, padrão para a cultivar. A área experimental foi localizada na maior região produtora de goiabas do Estado de São Paulo, no município de Vista Alegre do Alto, com coordenadas geográficas $21^{\circ} 08^{\prime}$ sul, $48^{\circ} 30^{\prime}$ oeste e altitude de $603 \mathrm{~m}$.

Segundo a classificação de Köppen, o clima local é do tipo Cwa subtropical com inverno curto, moderado e seco, verão quente e chuvoso, caracterizando duas estações distintas.

A diagnose foliar foi realizada pela amostragem de folhas ( $3^{\circ}$ par de folhas recém-maduras) no auge da floração, sendo coletados 12 pares de folhas por parcela (quatro pares em cada planta da parcela útil, sendo um par em cada quadrante da planta), na altura mediana das goiabeiras, seguindo as indicações de Natale et al. (1996). O material vegetal foi lavado em água destilada, seco em estufa com circulação de ar à temperatura de $65 \mathrm{a} 70^{\circ} \mathrm{C}$, até peso constante e moído para as determinações químicas dos teores de nutrientes, de acordo com a metodologia descrita por Bataglia et al. (1983).

A formação da base de dados para o desenvolvimento das normas DRIS foram os teores totais de nutrientes nas folhas em $\mathrm{g} \mathrm{kg}^{-1}$ para os macronutrientes e $\mathrm{mg} \mathrm{kg}^{-1}$ para os micronutrientes, e a produtividade das goiabeiras $\left(\mathrm{t} \mathrm{ha}^{-1}\right)$, avaliada nas seis safras.

Para o estabelecimento das normas DRIS, a base de dados foi dividida em duas subpopulações: uma com alta produtividade (ou população de referência) e outra de baixa produtividade. A população de alta produtividade foi estabelecida a partir de produtividades que fossem maiores que a produtividade média das áreas mais seu desvio-padrão $(\mathrm{m}+\mathrm{s})$, ou seja, acima de 73,2 tha ${ }^{-1}$. Ressalta-se, ainda, que em todas as safras acompanhadas havia parcelas com produções superiores à produtividade estabelecida como alta produtividade.

A média, o coeficiente de variação e a variância foram calculados para o grupo de referência ou alta produtividade e de baixa produtividade, utilizando as relações diretas e inversas.

Para calcular as funções das razões dos nutrientes, aplicou-se o método proposto por Jones (1981), a partir da seguinte equação:

$$
f\left(\frac{A}{B}\right)=\left[\left(\frac{A}{B_{a}}\right)-\left(\frac{A}{B_{r}}\right)\right] \cdot \frac{k}{s}
$$

Em que $A / B$ é a relação a cada dois nutrientes; $A / B_{a}$ é a relação a cada dois nutrientes da amostra que se deseja avaliar; $A / B_{r}$ é a relação a cada dois nutrientes da população de referência; $k$ é 
a constante de sensibilidade, e $s$ é o desvio-padrão da população de referência.

$\mathrm{O}$ valor de $\mathrm{k}=1$ foi utilizado no cálculo proposto.

Para o cálculo dos índices do DRIS, aplicou-se a fórmula geral proposta por Beaufils (1973), sendo para um nutriente $\mathrm{Y}$ :

$$
I Y=\frac{\sum f\left(\frac{A}{B}\right)-\sum f\left(\frac{A}{B}\right)}{n}
$$

Em que $n$ é número de funções DRIS analisadas.

O índice de balanço nutricional (IBN) foi calculado, somando-se os valores em módulo obtidos para cada nutriente:

$I B N=|I N|+|I P|+|I K|+|I C d+| I M g|+| I S|+| I B|+| I C u|+| I F e|+| I M n|+| I Z n \mid$

O índice de balanço nutricional médio $\left(\mathrm{IBN}_{\mathrm{m}}\right)$ foi calculado, somando-se os valores em módulo obtidos para cada nutriente e dividindo-o pelo número total de nutrientes (n) (WADT et al., 1998):

$I B N_{m}=\frac{|I N|+|I P|+|I K|+|I C q|+|I M g|+|I S|+|I B|+|I C z|+|I F e|+I M n|+| I Z n \mid}{n}$

A interpretação dos índices DRIS foi realizada tendo-se classificado os nutrientes de acordo com o potencial de resposta à adubação (PRA) (WADT, 2005). O PRA nulo é obtido quando o nutriente está em uma faixa de relativo equilíbrio nutricional, o que é refletido por seu valor em módulo inferior ao do IBNm. O PRA positivo ou nulo ocorre quando o nutriente se encontra em deficiência (índice negativo e superior, em módulo, ao do IBNm), mas sem que o nutriente apresente o menor índice DRIS, que é a condição para a ocorrência do PRA positivo. Essa distinção entre positiva ou nula e positiva ocorre em razão da probabilidade de resposta, que aumenta quando o índice é negativo e maior que o do IBNm, ser maior ainda quando o nutriente é o mais negativo. O PRA negativo é interpretado como excesso do nutriente, com índice DRIS positivo (superior ao do IBNm) e com maior valor entre os demais nutrientes.

Para maior síntese, as classes de potencial de resposta positiva (p) e positiva ou nula (pz) e, também, negativa (n) e negativa ou nula (nz) foram agrupadas e denominadas de limitantes por falta (LF) ou por excesso (LE). E a classe de resposta nula (z) foi denominada como não limitante (NL), conforme Silva et al. (2005).

Verificou-se a hipótese de que a frequência com que cada nutriente ocorreu como o limitante primário por deficiência, ou seja, com potencial de resposta à adubação positiva e de alta probabilidade, tenha sido atribuída ao acaso. Para isto, utilizou-se o teste de qui-quadrado de ajustamento (SILVA et al., 2005), a 5\% de probabilidade, com n - 1 graus de liberdade ( $\mathrm{n}=$ número de nutrientes analisados).

Considerou-se como o limitante primário por deficiência o nutriente que apresentou o índice negativo, de menor valor em relação aos índices dos demais nutrientes, e maior em módulo que o índice de equilíbrio nutricional médio $\left(\right.$ IEN $\left._{\mathrm{m}}\right)$. Se verdadeira a hipótese considerada, as frequências observadas para todos os nutrientes seriam estatisticamente iguais entre si. As frequências esperadas (FE) e observadas (FO) foram calculadas da seguinte forma (URANO et al., 2006):

$$
\begin{aligned}
& F E(\%)=\left(\frac{\frac{n^{\circ} \text { total de talhões avaliados }}{n^{\circ} \text { total de nutrientes avaliados }}}{n^{\circ} \text { total de talhões avaliados }}\right) \times 100 \\
& F O(\%)=\left(\frac{n^{\circ} \text { de talhões em que o mutriente foi }(p)}{n^{\circ} \text { de talhões avaliados }}\right) \times 100
\end{aligned}
$$

Para o estabelecimento dos níveis ótimos (ou nível crítico - NC) de cada nutriente, foi traçada a relação de cada nutriente com seu respectivo índice de balanço nutricional. Assim, as concentrações foliares de N, P, K, Ca, Mg, B, Cu, Fe, Mn e Zn adequadas para goaibeira 'Paluma' foram obtidas igualando-se todos os índices a zero na equação (y $=\mathrm{ax}+\mathrm{b}=0$ ), pois, teoricamente, quando todos esses índices tendem a zero, tem-se a condição ótima de equilíbrio nutricional da planta.

$\mathrm{O}$ limite inferior e o superior da faixa normal de concentração de nutrientes foram determinados de modo análogo ao utilizado por Serra et al. (2012), que consistiu em igualar os modelos estatísticos do relacionamento do teor de nutrientes e os índices DRIS a zero e $\pm 2 / 3$ do desvio-padrão (população de referência).

\section{RESULTADOS E DISCUSSÃO}

Das 168 amostras analisadas, 34 foram consideradas de alta produtividade ou subpopulação de referência (média + desvio-padrão), totalizando $20 \%$ da população. O restante $(80 \%)$ foi considerado a subpopulação de baixa produtividade.

As normas DRIS e seus respectivos desvios estão apresentadas na Tabela 1.

Para a diagnose realizada, rejeitou-se a hipótese de que as frequências dos diagnósticos, observa- 
das para todos os nutrientes, eram estatisticamente iguais entre si, o que indica que os métodos foram sensíveis para diagnosticar diferenças de probabilidade de resposta positiva à adubação, ou seja, com deficiência, para os nutrientes avaliados (Tabela 2).

Na Tabela 3, é apresentado o potencial de resposta à adubação. O nutriente com maior frequência de resposta à adubação (p) é o Fe, seguido pelo $\mathrm{K}$ e $\mathrm{Ca}$, respectivamente. $\mathrm{O}$ nutriente que mais está em excesso ou baixa resposta à adubação (n) seria o $\mathrm{Cu}$. Ainda, nos mesmos dados, procedeu-se à análise da classe do estado nutricional, segundo Silva et al. (2005), cujos resultados estão apresentados na Tabela 4.

A ordem dos nutrientes com maior frequência de limitação por falta, em ordem decrescente, é: $\mathrm{Fe}>\mathrm{K}>\mathrm{Mn}>\mathrm{Ca}>\mathrm{B}>\mathrm{N}>\mathrm{P}>\mathrm{Cu}>\mathrm{S}>\mathrm{Mg}>\mathrm{Zn}$, e commaior frequência de limitação por excesso, em ordem decrescente, é: $\mathrm{Cu}>\mathrm{Fe}>\mathrm{P}>\mathrm{Mn}=\mathrm{Mg}>\mathrm{Zn}>\mathrm{Ca}>\mathrm{B}>\mathrm{K}>\mathrm{S}>\mathrm{N}$.

A justificativa para o cobre ser o nutriente com maior limitação por excesso pode ser devido aos tratamentos fitossanitários. Em relação ao ferro, nutriente com maior limitação por falta, Bataglia et al. (2004) citam que nutrientes como o Fe e o Mn apresentam grande variabilidade de concentrações devido a condições edafoclimátivas.

Destaca-se que, entre os macronutrientes, o P é o nutriente com mais casos limitantes por excesso. Ressalta-se, também, que a goiabeira adulta não apresenta grandes respostas à aplicação de $\mathrm{P}$ (NATALE et al., 2001). O resultado referente ao cobre (excesso) pode ser em função das aplicações de defensivos realizadas no pomar.

$\mathrm{Na}$ Índia, em estudo para estabelecer normas DRIS, Hundal et al. (2007) verificaram que 8; 5; $4 ; 14 ; 12 ; 7 ; 17 ; 12$ e $17 \%$ das amostras de folhas de goiabeiras avaliadas em Punjab estavam com níveis altos de $\mathrm{N}, \mathrm{P}, \mathrm{K}, \mathrm{Ca}, \mathrm{Mg}, \mathrm{S}, \mathrm{Mn}, \mathrm{Zn}$ e $\mathrm{Cu}$, respectivamente, ou seja, as condições de cultivo e principalmente de solo retratam realidades diferentes quanto à nutrição da planta.

Considerando a classe do estado nutricional (SILVA et al., 2005), para efeitos de comparação entre o método DRIS e as classes de interpretação de Natale et al. (1996), utilizada como referência, constata-se que os nutrientes que obtiveram maior número de casos concordantes, em ordem decrescente, foram: $\mathrm{Fe}>\mathrm{S}>\mathrm{P}>\mathrm{N}>\mathrm{Ca}>\mathrm{Mn}>\mathrm{Cu}>\mathrm{Zn}>\mathrm{K}>\mathrm{B}>\mathrm{Mg}$. A maior parte dos diagnósticos concordantes esteve relacionada às avaliações dos nutrientes não limitantes, seguido das avaliações dos nutrientes limitantes por excesso e, finalmente, dos limitantes por falta (Tabela 5).

O DRIS foi desenvolvido para tornar a interpretação menos dependente de variações de amostragens com respeito à idade e à origem do tecido vegetal, para permitir um ordenamento de nutrientes limitantes ao crescimento e para realçar a importância do equilíbrio nutricional da planta (BATAGLIA e SANTOS, 1990), permitindo, assim, um uso universal das normas DRIS. No entanto, Reis e Monnerat (2002) realizam raciocínio contrário ao citado por Bataglia e Santos (1990).

$\mathrm{Na}$ Figura1, verifica-se que os valores de IBNm obtidos apresentaram significância, com ajuste da equação linear quando relacionados à produção.

A regressão entre o IBNm e a produtividade

(Figura 1) indica que a produção de frutos esteve significativamente associada ao estado nutricional das plantas $\left(\mathrm{R}^{2}=0,42\right.$ e $\left.\mathrm{p}<0,01\right)$.

Valores de IBN baixos são indicadores de plantas bem nutridas e corresponderam às amostras de plantas com maior produtividade, ratificando a importância do balanço nutricional para a produção de frutos (TEIXEIRA et al., 2007).

Foram ajustadas equações de regressão para a relação entre os teores de nutrientes em folhas de goiabeiras e os índices DRIS (Tabela 6).

Realizou-se a comparação entre as faixas de suficiência indicadas por Natale et al. (1996) e Quaggio et al. (1997) para goiabeiras, e as faixas geradas no presente estudo. Na Tabela 7, são apresentadas as faixas adequadas, observando-se que houve diferenças nas amplitudes.

Ressalta-se que os manejos inerentes à cultura da goiaba se modificaram quando comparados aos trabalhos de adubação realizados por Natale et al. (1995) e Natale et al. (1996a), sendo que boa parte dos pomares na região de Taquaritinga, Monte Alto e Vista Alegre do Alto é irrigada, com utilização da poda drástica e adubação constante, permitindo altas produções e com possibilidade de até 3 safras em 2 anos (SOUZA et al., 2012; ROZANE et al., 2009).

Hundal et al. (2007) derivaram faixas de suficiência de normas DRIS para goiabeiras na Índia, cujos valores foram 14,1-16,5; 1,0-1,7; 5,1-9,7; 11,621,2; 3,1-5, 1 e 1,8-2,8 $\left(\mathrm{g} \mathrm{kg}^{-1}\right)$ para N, P, K, Ca, Mg e $\mathrm{S}$, e $105-153$; 58-110; 5-29 e 6-16 (mg kg-1) para $\mathrm{Fe}, \mathrm{Mn}, \mathrm{Zn}$ e $\mathrm{Cu}$, respectivamente. Assim, observa-se a grande diferença das faixas geradas no presente estudo, em condições e solo tropical, e as da Índia, país que também é um dos maiores produtores da fruta no mundo.

Entre os macronutrientes, a maior diferença entre os padrões da literatura e o teor estimado a partir do DRIS foi em relação ao $\mathrm{Mg}$. Indicando que como verificado por Natale et al. (2007) no solo, que o valor de saturação por bases de $50 \%$, ou seja, valor menor 
do recomendado por Santos e Quaggio (1997), seja satisfatório, afinal a saturação por bases está diretamente correlacionada ao teor de $\mathrm{Ca}$ e $\mathrm{Mg}$ nas folhas.

Entre os micronutrientes, verifica-se que os teores de $\mathrm{Cu}, \mathrm{Fe}$ e $\mathrm{Mn}$ apresentaram maiores amplitudes em relação a Natale et al. (1996).

Em trabalho avaliando normas DRIS em pomares de goiabeiras, na região de Punjab (Índia), Hundal et al. (2007) verificaram que mais de 50\% das amostras avaliadas apresentavam deficiência de N, 2\% de P, 15\% de Mn, 6\% de $\mathrm{Zn}$ e $7 \%$ de $\mathrm{Cu}$. Os autores ainda citam que, baseado nos índices obtidos com o uso do DRIS, na avaliação da fertilidade do solo e no manejo da adubação, cada nutriente poderia ser efetivamente aplicado.

Teixeira et al. (2007) avaliaram normas DRIS e faixas de suficiência para bananeiras, no Estado de São Paulo, e verificaram que os valores gerados pelo DRIS foram próximos aos relatados na literatura.

Nachtigall e Dechen (2007) citam que o método DRIS apresentou desempenho semelhante ao critério de faixa de suficiência e pode ser utilizado para a interpretação da análise foliar em macieira, por apresentar valores de IBN que indicam o estado nutricional das plantas e pela eficiência no diagnóstico nutricional da cultura.

TABELA 1 - Média e desvio- padrão das relações na subpopulação de alta produtividade

\begin{tabular}{|c|c|c|c|c|c|c|c|c|c|c|}
\hline Relação & $\mathbf{N} / \mathbf{P}$ & $\mathbf{N} / \mathbf{K}$ & N/CA & N/Mg & $\mathbf{N} / \mathbf{S}$ & N/B & $\mathbf{N} / \mathbf{C} \mathbf{U}$ & $\mathrm{N} / \mathrm{Fe}$ & N/Mn & $N / Z n$ \\
\hline Norma (x) & 12,18 & 1,24 & 2,11 & 9,09 & 7,29 & 0,68 & 1,32 & 0,30 & 0,28 & 0,99 \\
\hline Desvio Padrão & 1,17 & 0,18 & 0,31 & 1,48 & 1,35 & 0,20 & 0,60 & 0,13 & 0,08 & 0,29 \\
\hline Relação & $\mathbf{P} / \mathbf{N}$ & $\mathbf{P} / \mathbf{K}$ & $\mathbf{P} / \mathbf{C a}$ & $\mathrm{P} / \mathrm{Mg}$ & $\mathbf{P} / \mathbf{S}$ & $\mathbf{P} / \mathbf{B}$ & $\mathrm{P} / \mathrm{Cu}$ & $\mathbf{P} / \mathbf{F e}$ & P/Mn & $\mathbf{P} / \mathbf{Z n}$ \\
\hline Norma (x) & 0,08 & 0,10 & 0,17 & 0,75 & 0,60 & 0,06 & 0,11 & 0,02 & 0,02 & 0,08 \\
\hline Desvio- Padrão & 0,01 & 0,02 & 0,03 & 0,11 & 0,10 & 0,02 & 0,05 & 0,01 & 0,01 & 0,02 \\
\hline Relação & $\mathbf{K} / \mathbf{N}$ & $\mathbf{K} / \mathbf{P}$ & $\mathrm{K} / \mathrm{Ca}$ & $\mathrm{K} / \mathrm{Mg}$ & $\mathbf{K} / \mathbf{S}$ & K/B & $\mathrm{K} / \mathrm{Cu}$ & $\mathrm{K} / \mathrm{Fe}$ & K/Mn & $\mathbf{K} / \mathbf{Z n}$ \\
\hline Norma (x) & 0,82 & 9,99 & 1,73 & 7,53 & 5,90 & 0,54 & 1,11 & 0,25 & 0,23 & 0,82 \\
\hline Desvio- Padrão & 0,12 & 1,65 & 0,34 & 1,81 & 1,00 & 0,12 & 0,56 & 0,14 & 0,06 & 0,28 \\
\hline Relação & $\mathrm{Ca} / \mathrm{N}$ & $\mathrm{Ca} / \mathrm{P}$ & $\mathrm{Ca} / \mathrm{K}$ & $\mathrm{Ca} / \mathrm{Mg}$ & $\mathrm{Ca} / \mathrm{S}$ & $\mathrm{Ca} / \mathrm{B}$ & $\mathrm{Ca} / \mathrm{Cu}$ & $\mathrm{Ca} / \mathrm{Fe}$ & $\mathrm{Ca} / \mathrm{Mn}$ & $\mathrm{Ca} / \mathrm{Zn}$ \\
\hline Norma (x) & 0,49 & 5,88 & 0,61 & 4,33 & 3,51 & 0,33 & 0,64 & 0,14 & 0,14 & 0,48 \\
\hline Desvio-Padrã & 0,08 & 0,90 & 0,15 & 0,52 & 0,74 & 0,10 & 0,31 & 0,06 & 0,05 & 0,15 \\
\hline Relação & $\mathbf{M g} / \mathbf{N}$ & $\mathbf{M g} / \mathbf{P}$ & $\mathrm{Mg} / \mathrm{K}$ & $\mathrm{Mg} / \mathrm{Ca}$ & $\mathrm{Mg} / \mathrm{S}$ & $\mathrm{Mg} / \mathrm{B}$ & $\mathrm{Mg} / \mathrm{Cu}$ & $\mathbf{M g} / \mathbf{F e}$ & Mg/Mn & $\mathrm{Mg} / \mathrm{Zn}$ \\
\hline Norma (x) & 0,11 & 1,37 & 0,14 & 0,23 & 0,82 & 0,08 & 0,15 & 0,03 & 0,03 & 0,11 \\
\hline Desvio- Padrão & 0,02 & 0,23 & 0,04 & 0,03 & 0,21 & 0,03 & 0,07 & 0,01 & 0,01 & 0,04 \\
\hline Relação & $\mathbf{S} / \mathbf{N}$ & $\mathbf{S} / \mathbf{P}$ & $\mathbf{S} / \mathbf{K}$ & $\mathrm{S} / \mathrm{Ca}$ & S/Mg & S/B & $\mathrm{S} / \mathrm{Cu}$ & $\mathrm{S} / \mathrm{Fe}$ & S/Mn & $\mathbf{S} / \mathbf{Z n}$ \\
\hline Norm & 0,14 & 1,71 & & & 1,29 & 0,09 & 20 & 0,04 & 0,04 & 0,14 \\
\hline Desvio- Padrão & 0,03 & 0,28 & 0,03 & 0,06 & 0,32 & 0,02 & 0,10 & 0,02 & 0,01 & 0,05 \\
\hline Relação & $\mathbf{B} / \mathbf{N}$ & $\mathbf{B} / \mathbf{P}$ & $\mathbf{B} / \mathbf{K}$ & $\mathrm{B} / \mathrm{Ca}$ & $\mathrm{B} / \mathrm{Mg}$ & $\mathbf{B} / \mathbf{S}$ & $\mathrm{B} / \mathrm{CU}$ & $\mathrm{B} / \mathrm{Fe}$ & B/Mn & $\mathbf{B} / \mathbf{Z n}$ \\
\hline Norma (x) & 1,61 & 19,48 & 1,93 & 3,36 & 14,83 & 11,26 & 2,27 & 0,52 & 0,45 & 1,62 \\
\hline Desvio- Padrão & 0,53 & 6,24 & 0,42 & 1,11 & 5,93 & 2,58 & 1,40 & 0,37 & 0,17 & 0,79 \\
\hline Relação & $\mathrm{Cu} / \mathrm{N}$ & $\mathbf{C u} / \mathbf{P}$ & $\mathrm{Cu} / \mathrm{K}$ & $\mathrm{Cu} / \mathrm{Ca}$ & $\mathrm{Cu} / \mathrm{Mg}$ & & $\mathrm{Cu} / \mathrm{B}$ & $\mathrm{Cu} / \mathrm{Fe}$ & $\mathrm{Cu} / \mathrm{Mn}$ & $\mathrm{Cu} / \mathrm{Zn}$ \\
\hline Norma (x) & 1,47 & 19,01 & 1,90 & 3,13 & 13,82 & 12,44 & 1,14 & 0,38 & 0,39 & 1,42 \\
\hline Desvio- Padrão & 1,70 & 23,54 & 2,27 & 3,67 & 16,80 & 16,73 & 1,50 & 0,40 & 0,44 & 1,62 \\
\hline Relação & $\mathrm{Fe} / \mathrm{N}$ & $\mathrm{Fe} / \mathrm{P}$ & $\mathrm{Fe} / \mathrm{K}$ & $\mathrm{Fe} / \mathrm{Ca}$ & $\mathrm{Fe} / \mathrm{Mg}$ & $\mathrm{Fe} / \mathrm{S}$ & $\mathrm{Fe} / \mathrm{B}$ & $\mathrm{Fe} / \mathrm{Cu}$ & $\mathrm{Fe} / \mathrm{Mn}$ & $\mathrm{Fe} / \mathrm{Zn}$ \\
\hline Norma (x) & 4,20 & 50,16 & 5,48 & 8,55 & 35,83 & 31,04 & 3,08 & 5,36 & 1,23 & 3,90 \\
\hline Desvio- Padrão & 2,12 & 22,53 & 3,34 & 3,57 & 12,82 & 16,16 & 1,97 & 3,67 & 0,85 & 2,07 \\
\hline Relação & $\mathbf{M n} / \mathbf{N}$ & $\mathbf{M n} / \mathbf{P}$ & $\mathbf{M n} / \mathbf{K}$ & $\mathrm{Mn} / \mathrm{Ca}$ & $\mathbf{M n} / \mathbf{M g}$ & $\mathbf{M n} / \mathbf{S}$ & $\mathbf{M n} / \mathbf{B}$ & $\mathrm{Mn} / \mathrm{Cu}$ & $\mathrm{Mn} / \mathrm{Fe}$ & $\mathbf{M n} / \mathbf{Z n}$ \\
\hline Norma (x) & 3,91 & 48,25 & 4,84 & 8,41 & 36,25 & 28,67 & 2,75 & 4,92 & 1,14 & 3,83 \\
\hline Desvio -Padrão & 1,53 & 21,49 & 2,01 & 4,17 & 17,36 & 13,56 & 1,76 & 2,62 & 0,55 & 1,62 \\
\hline Relação & $\mathrm{Zn} / \mathbf{N}$ & $\mathrm{Zn} / \mathbf{P}$ & $\mathrm{Zn} / \mathrm{K}$ & $\mathrm{Zn} / \mathrm{Ca}$ & $\mathrm{Zn} / \mathrm{Mg}$ & $\mathrm{Zn} / \mathrm{S}$ & $\mathrm{Zn} / \mathrm{B}$ & $\mathrm{Zn} / \mathrm{Cu}$ & $\mathrm{Zn} / \mathrm{Fe}$ & $\mathbf{Z n} / \mathbf{M n}$ \\
\hline Norma (x) & 1,15 & 13,78 & 1,44 & 2,36 & 10,12 & 8,27 & 0,79 & 1,50 & 0,31 & 0,33 \\
\hline Desvio- Padrão & 0,58 & 5,89 & 0,81 & 0,92 & 3,80 & 3,79 & 0,44 & 0,97 & 0,13 & 0,22 \\
\hline
\end{tabular}


TABELA 2- Cálculo do qui-quadrado, para as frequências observadas (\%) de N, P, K, Ca, Mg, S, B, Cu, $\mathrm{Fe}, \mathrm{Mn}$ e Zn como nutriente limitante primário por deficiência ${ }^{(1)}$, na suposição de que as frequências observadas são consequência do acaso, em amostras de folhas coletadas em pomar de goiabeiras, na subpopulação de baixa produtividade ${ }^{(2)}$.

\begin{tabular}{cccc}
\hline Nutriente & $\mathbf{F O}^{(3)}$ & $\mathbf{F E}^{(3)}$ & $\mathbf{F O - F E} / \mathbf{F E}^{(3)}$ \\
\hline $\mathbf{N}$ & 6,72 & 11 & $-0,39$ \\
$\mathbf{P}$ & 4,48 & 11 & $-0,59$ \\
$\mathbf{K}$ & 10,45 & 11 & $-0,05$ \\
$\mathbf{C a}$ & 7,46 & 11 & $-0,32$ \\
$\mathbf{M g}$ & 0,75 & 11 & $-0,93$ \\
$\mathbf{S}$ & 8,21 & 11 & $-0,25$ \\
$\mathbf{B}$ & 4,48 & 11 & $-0,59$ \\
$\mathbf{C u}$ & 1,49 & 11 & $-0,86$ \\
$\mathbf{F e}$ & 46,27 & 11 & 3,21 \\
$\mathbf{M n}$ & 8,21 & 11 & $-0,25$ \\
$\mathbf{Z n}$ & 0,00 & 11 & $-1,00$ \\
\hline & & Qui-quadrado & $153,83 * *$ \\
\hline
\end{tabular}

${ }^{(1)}$ Com potencial de resposta à adubação positiva e muito provável (p), conforme Wadt (2005). ${ }^{(2)}$ Menor que a média + desvio-padrão. ${ }^{(3)} \mathrm{FO}$ e FE correspondem às frequências observada e esperada, respectivamente. ** Significativo a $1 \%$.

TABELA 3- Frequência (\%) do potencial de resposta à adubação de $\mathrm{N}, \mathrm{P}, \mathrm{K}, \mathrm{Ca}, \mathrm{Mg}, \mathrm{S}, \mathrm{B}, \mathrm{Cu}, \mathrm{Fe}, \mathrm{Mn}$ e Zn, em amostras de folhas coletadas em pomar de goiabeiras, na subpopulação de baixa produtividade $^{(1)}$

\begin{tabular}{cccccc}
\hline Nutriente & $\mathbf{p}$ & $\mathbf{p z}$ & $\mathbf{z}$ & $\mathbf{n z}$ & $\mathbf{n}$ \\
\hline $\mathbf{N}$ & 6,7 & 12,7 & 76,1 & 4,5 & 0,0 \\
$\mathbf{P}$ & 4,5 & 8,2 & 63,4 & 15,7 & 8,2 \\
$\mathbf{K}$ & 10,4 & 23,1 & 59,7 & 4,5 & 2,2 \\
$\mathbf{C a}$ & 7,5 & 17,2 & 63,4 & 11,9 & 0,0 \\
$\mathbf{M g}$ & 0,7 & 5,2 & 70,9 & 17,2 & 6,0 \\
$\mathbf{S}$ & 8,2 & 2,2 & 84,3 & 4,5 & 0,7 \\
$\mathbf{B}$ & 4,5 & 15,7 & 70,9 & 3,7 & 5,2 \\
$\mathbf{C u}$ & 1,5 & 9,7 & 31,3 & 2,2 & 55,2 \\
$\mathbf{F e}$ & 46,3 & 1,5 & 26,9 & 14,9 & 10,4 \\
$\mathbf{M n}$ & 8,2 & 18,7 & 50,0 & 20,1 & 3,0 \\
$\mathbf{Z n}$ & 0,0 & 5,2 & 73,9 & 12,7 & 8,2 \\
\hline
\end{tabular}

${ }^{(1)}$ Menor que a média + desvio-padrão. ${ }^{(2)}$ p: positiva, com alta probabilidade; pz: positiva, com baixa probabilidade; z: nula; nz: negativa, com baixa probabilidade; n: negativa, com alta probabilidade, conforme Wadt (2005).

TABELA 4- Frequência (\%) das classes de resposta para os nutrientes N, P, K, Ca, Mg, S, B, Cu, Fe, Mn e $\mathrm{Zn}$ em amostras de folhas coletadas, em pomar de goiabeiras, na subpopulação de baixa produtividade $^{(1)}$

\begin{tabular}{ccccc}
\hline Nutrientes & $\mathbf{L F}^{(2)}$ & $\mathbf{N L}$ & $\mathbf{L E}$ & Total \\
\hline $\mathbf{N}$ & 19,4 & 76,1 & 4,5 & 100 \\
$\mathbf{P}$ & 12,7 & 63,4 & 23,9 & 100 \\
$\mathbf{K}$ & 33,6 & 59,7 & 6,7 & 100 \\
$\mathbf{C a}$ & 24,6 & 63,4 & 11,9 & 100 \\
$\mathbf{M g}$ & 6,0 & 70,9 & 23,1 & 100 \\
$\mathbf{S}$ & 10,4 & 84,3 & 5,2 & 100 \\
$\mathbf{B}$ & 20,1 & 70,9 & 9,0 & 100 \\
$\mathbf{C u}$ & 11,2 & 31,3 & 57,5 & 100 \\
$\mathbf{F e}$ & 47,8 & 26,9 & 25,4 & 100 \\
$\mathbf{M n}$ & 26,9 & 50,0 & 23,1 & 100 \\
$\mathbf{Z n}$ & 5,2 & 73,9 & 20,9 & 100 \\
\hline
\end{tabular}

(1) Menor que a média + desvio-padrão. ${ }^{(2)}$ LF - limitante por falta; NL - não limitante; LE - limitante por excesso. 
TABELA 5- Frequência (\%) de diagnósticos concordantes para a avaliação do estado nutricional pelo método DRIS e teores adequados de Natale et al. (1996) para N, P, K, Ca, Mg, S, B, Cu, Fe, Mn e Zn, em amostras de folhas coletadas, em pomar de goiabeiras, na subpopulação de baixa produtividade $^{(1)}$

\begin{tabular}{ccccc}
\hline Nutrientes & LF & NL & LE & $\begin{array}{c}\text { Valores } \\
\text { médios }\end{array}$ \\
\hline \multicolumn{4}{c}{-------------- \% } & ------------ \\
\hline $\mathbf{N}$ & 54,2 & 66,7 & 33,3 & 51,4 \\
$\mathbf{P}$ & 29,4 & 81,2 & 53,3 & 54,6 \\
$\mathbf{C a}$ & 2,2 & 74,8 & 34,6 & 37,2 \\
$\mathbf{M g}$ & 6,1 & 81,7 & 57,1 & 48,3 \\
$\mathbf{S}$ & 6,3 & 5,3 & 3,2 & 4,9 \\
$\mathbf{B}$ & 100,0 & 95,8 & 28,6 & 74,8 \\
$\mathbf{C u}$ & 0,0 & 16,8 & 10,2 & 9,0 \\
$\mathbf{F e}$ & 27,3 & 4,8 & 100,0 & 44,0 \\
$\mathbf{M n}$ & 100,0 & 72,2 & 77,3 & 83,2 \\
$\mathbf{Z n}$ & 0,0 & 80,7 & 60,8 & 47,2 \\
& 13,0 & 67,7 & 46,4 & 42,4 \\
\hline
\end{tabular}

${ }^{(1)}$ Menor que a média + desvio-padrão. ${ }^{(2)}$ LF - limitante por falta; NL - não limitante; LE - limitante por excesso.

TABELA 6- Modelos estatísticos dos relacionamentos entre os teores de nutrientes e os índices DRIS, em amostras de folhas, em pomar de goiabeiras.

\begin{tabular}{cc}
\hline Nutriente & Equação \\
\hline $\mathbf{N}$ & $\mathrm{IN}=1,3743(\mathrm{~N})-26,633^{* *}$ \\
$\mathbf{P}$ & $\mathrm{IP}=21,171(\mathrm{P})-33,853^{* *}$ \\
$\mathbf{K}$ & $\mathrm{IK}=5,394(\mathrm{~K})-88,176^{* *}$ \\
$\mathbf{C a}$ & $\mathrm{ICa}=4,4185(\mathrm{Ca})-41,417^{* *}$ \\
$\mathbf{M g}$ & $\mathrm{IMg}=20,045(\mathrm{Mg})-44,158^{* *}$ \\
$\mathbf{S}$ & $\mathrm{IS}=28,514(\mathrm{~S})-77,055^{* *}$ \\
$\mathbf{B}$ & $\mathrm{IB}=2,3127(\mathrm{~B})-69,838^{* *}$ \\
$\mathbf{C u}$ & $\mathrm{ICu}=0,4668(\mathrm{Cu})-14,457^{* *}$ \\
$\mathbf{F e}$ & $\mathrm{IFe}=0,3833(\mathrm{Fe})-31,845^{* *}$ \\
$\mathbf{M n}$ & $\mathrm{IMn}=0,3898(\mathrm{Mn})-29,927^{* *}$ \\
$\mathbf{Z n}$ & $\mathrm{IZn}=1,3686(\mathrm{Zn})-26,926^{* *}$ \\
\hline
\end{tabular}

** - Significativo a $1 \%$.

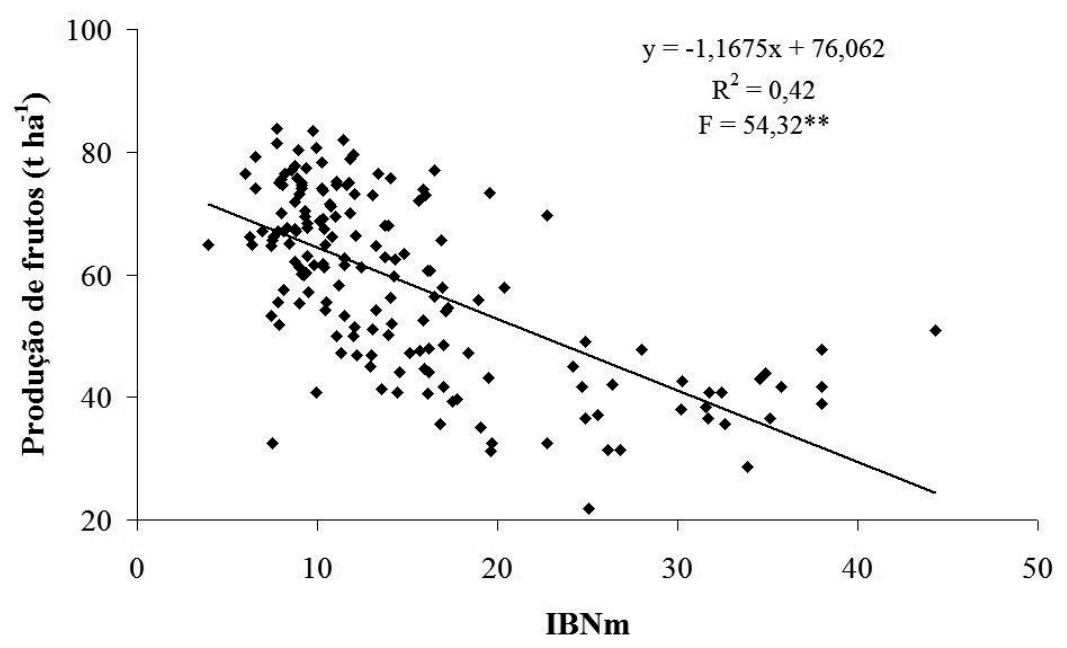

FIGURA 1- Relação entre os valores de IBN e a produção de frutos em pomar de goiabeira. ** - Significativo a $1 \%$. 
TABELA 7- Faixa normal de nutrientes em amostras de folhas coletadas, em pomar de goiabeiras, obtidas pelos métodos DRIS e da literatura utilizada para a comparação dos valores ${ }^{(1)}$

\begin{tabular}{|c|c|c|c|}
\hline Nutriente & Método & Faixa adequada & $\begin{array}{c}\text { Teor } \\
\text { adequado }\end{array}$ \\
\hline \multirow{3}{*}{$\mathbf{N}$} & DRIS & $18-21$ & 19 \\
\hline & Natale et al. (1996) & $20-23$ & - \\
\hline & Quaggio et al. (1997) & $13-16$ & - \\
\hline \multirow{3}{*}{$\mathbf{P}$} & DRIS & $1,5-1,7$ & 1,6 \\
\hline & Natale et al. (1996) & $1,4-1,8$ & - \\
\hline & Quaggio et al. (1997) & $1,4-1,6$ & - \\
\hline \multirow{3}{*}{$\mathbf{K}$} & DRIS & $15-17$ & 16 \\
\hline & Natale et al. (1996) & $14-17$ & - \\
\hline & Quaggio et al. (1997) & $13-16$ & - \\
\hline \multirow{3}{*}{$\mathbf{C a}$} & DRIS & $8-11$ & 9 \\
\hline & Natale et al. (1996) & $7-11$ & - \\
\hline & Quaggio et al. (1997) & $9-15$ & - \\
\hline \multirow{3}{*}{ Mg } & DRIS & $1,8-2,5$ & 2,2 \\
\hline & Natale et al. (1996) & $3,4-4,0$ & - \\
\hline & Quaggio et al. (1997) & $2,4-4,0$ & - \\
\hline \multirow{3}{*}{$\mathbf{S}$} & DRIS & $2,5-2,9$ & 2,7 \\
\hline & Natale et al. (1996) & $2,5-3,5$ & - \\
\hline & Quaggio et al. (1997) & - & - \\
\hline \multirow{3}{*}{ B } & DRIS & $26-38$ & 30 \\
\hline & Natale et al. (1996) & $20-25$ & - \\
\hline & Quaggio et al. (1997) & - & - \\
\hline \multirow{3}{*}{$\mathrm{Cu}$} & DRIS & $5-57$ & 31 \\
\hline & Natale et al. (1996) & $20-40$ & - \\
\hline & Quaggio et al. (1997) & - & - \\
\hline \multirow{3}{*}{$\mathbf{F e}$} & DRIS & $54-112$ & 83 \\
\hline & Natale et al. (1996) & $60-90$ & - \\
\hline & Quaggio et al. (1997) & - & - \\
\hline \multirow{3}{*}{ Mn } & DRIS & $53-101$ & 77 \\
\hline & Natale et al. (1996) & $40-80$ & - \\
\hline & Quaggio et al. (1997) & - & - \\
\hline \multirow{3}{*}{ Zn } & DRIS & $13-26$ & 20 \\
\hline & Natale et al. (1996) & $25-35$ & - \\
\hline & Quaggio et al. (1997) & - & - \\
\hline
\end{tabular}

${ }^{(1)}$ Faixa ótima estimada a partir dos limites inferior e superior, igualando a zero as equações de relacionamento de teores de nutrientes e índices DRIS e $\pm 2 / 3$ do desvio- padrão, de acordo com Serra et al. (2012).

\section{CONCLUSÕES}

1-Aordem dos nutrientes com maior frequência de limitação por falta, em ordem decrescente, seria: $\mathrm{Fe}>\mathrm{K}>\mathrm{Mn}>\mathrm{Ca}>\mathrm{B}>\mathrm{N}>\mathrm{P}>\mathrm{Cu}>\mathrm{S}>\mathrm{Mg}>\mathrm{Zn}$, e limitantes por excesso, em ordem decrescente, seria: $\mathrm{Cu}>\mathrm{Fe}>\mathrm{P}>\mathrm{Mn}=\mathrm{Mg}>\mathrm{Zn}>\mathrm{Ca}>\mathrm{B}>\mathrm{K}>\mathrm{S}>\mathrm{N}$.
2-As faixas adequadas provenientes dos índices DRIS são: 18-21; 1,5-1,7; 15-17; 8-11; 1,82,5 e 2,5-2,9 $\left(\mathrm{g} \mathrm{kg}^{-1}\right)$, para os macronutrientes $\mathrm{N}, \mathrm{P}, \mathrm{K}$, $\mathrm{Ca}, \mathrm{Mg}$ e S, respectivamente, e 26-38; 5-57; 54-112; 53-101 e 13-126 (mg kg-1) para os micronutrientes $\mathrm{B}, \mathrm{Cu}, \mathrm{Fe}, \mathrm{Mn}$ e $\mathrm{Zn}$.

3-A produtividade das goiabeiras esteve associada ao estado nutricional. 


\section{AGRADECIMENTOS}

À indústria de Polpas e Conservas Ltda., pelo auxílio na condução do ensaio.

\section{REFERÊNCIAS}

BATAGLIA, O. C.; FURLANI, A. M. C.; TEIXEIRA, J. P. F.; FURLANI, P. R.; GALLO, J. R. Métodos de análise química de plantas. Campinas: Instituto Agronômico, 1983. 48 p. (Boletim técnico, 78).

BATAGLIA, O. C.; QUAGGIO, J. A.; SANTOS, W. R.; ABREU, M. F. Diagnose nutricional do cafeeiro pelo DRIS variando-se a constante de sensibilidade dos nutrientes de acordo com a intensidade de frequência de resposta na produção. Bragantia, Campinas, v. 63, p. 253-263, 2004.

BATAGLIA, O. C.; SANTOS, W. R. Efeito do procedimento de cálculo e da população de referência nos índices do DRIS. Revista Brasileira de Ciência do Solo, Viçosa, v. 14, p. 334-339, 1990.

BEAUFILS, E. R. Diagnosis and recommendation integrated system (DRIS). A general scheme for experimentation and calibration based on principles developed from research in plant nutrition. Pietermararitzburg: University of Natal, 1973. 132p. (Soil Science Bulletin, 1).

CRESTE, J. E. Perspectivas do DRIS em culturas de alta produtividade. In: PRADO, R. M.; ROZANE, D. E.; VALE, D. W.; CORREIA, M. A. R.; SOUZA, H. A. Nutrição de plantas aplicada a sistemas de alta produtividade. Jaboticabal: Capes/Fundunesp, 2008. p. 83-104.

DIAS, J. R. M.; PEREZ, D. V.; SILVA, L. M.; LEMOS, C. O.; WADT, P. G. S. Normas DRIS para cupuaçuzeiro cultivado em monocultivo e em sistemas agroflorestais. Pesquisa Agropecuária Brasileira, Brasília, v. 45, p. 64-71, 2010.

HUNDAL, H. S.; SINGH, D.; SINGH, K. Trees in Punjab, northwest India through the diagnostic and recommendation integrated system approach. Communication in Soil Science and Plant Analysis, New York, v. 38, p. 2.117-2.130, 2007.

JONES, C.A. Proposed modifications of the diagnosis and recommendation integrated system (DRIS) for interpreting plant analyses. Communication in Soil Science and Plant Analysis, New York, v. 12, p. 785-794, 1981.
MALAVOLTA, E. Manual de nutrição mineral de plantas. São Paulo: Editora Agronômica Ceres, 2006. $638 \mathrm{p}$.

MOURÃO FILHO, F. A. A. DRIS and sufficient range approaches in nutritional diagnosis of "Valencia" sweet orange on three rootstocks. Journal of Plant Nutrition, New York, v. 28, p. 691-705, 2005.

MOURÃO FILHO, F. A. A.; AZEVEDO, J. C. DRIS normas for 'Valência' sweet orange on three rootstocks. Pesquisa Agropecuária Brasileira, Brasília, v. 38, p. 85-93, 2005.

NATALE, W.; COUTINHO, E. L. M.; BOARETTO, A. E.; CENTURION, J. F. Resposta da goiabeira (Psidium guajava L.) cv. Paluma em formação à adubação fosfatada. Revista Brasileira de Fruticultura, Jaboticabal, v. 23, p. 92-96, 2001.

NATALE, W.; COUTINHO, E. L. M.; BOARETTO, A. E.; PEREIRA, F. M. Goiabeira: calagem e adubação. Jaboticabal: FUNEP, 1996b. 22 p.

NATALE, W.; COUTINHO, E. L. M. ; PEREIRA, F. M.; BOARETTO, A. E.; OIOLI, A. A. P.; SALES, L. Adubação nitrogenada na cultura da goiabeira. Revista Brasileira de Fruticultura, Jaboticabal, v. 17, p. 7-15, 1995.

NATALE, W.; COUTINHO, E. L. M. ; PEREIRA, F. M.; OIOLI, A. A. P.; SALES, L. Nutrição e adubação potássica na cultura da goiabeira. Revista Brasileira de Ciência do Solo, Viçosa, v. 20, p. 247-250, 1996a.

NATALE, W.; PRADO, R. M.; ROZANE, D. E.; ROMUALDO, L. M. Efeitos da calagem na fertilidade do solo e na nutrição e produtividade da goiabeira. Revista Brasileira de Ciência do Solo, Viçosa, v. 31, p. 1475-1485, 2007.

NACHTIGALL, G. R.; DECHEN, A. R. DRIS como método diagnóstico de nutrição mineral de plantas. In: PRADO, R. M.; CECÍLIO FILHO, A. B.; CORREIA, M. A. R.; PUGA, A. P. Nutrição de plantas: diagnose foliar em hortaliças. Jaboticabal: FCAV,FAPESP, 2010. p. 185-198.

NACHTIGALL, G. R.; DECHEN, A. R. DRIS norms evaluating the nutritional state of apple tree. Scientia Agricola, Piracicaba, v. 64, p. 282-287, 2007. 
POLITI, L. S.; FLORES, R. A.; SILVA, J. A. S.; WADT, P. G. S.; PINTO, P. A. C.; PRADO, R. M. Estado nutricional de mangueiras determinado pelos métodos DRIS e CND. Revista Brasileira de Engenharia Agrícola e Ambiental, Campina Grande, v. 17, p. 11-18, 2013.

QUAGGIO, J. A.; RAIJ, B. van; PIZA JÚNIOR, C. T. Frutíferas. In: IAC. Recomendações de adubação e calagem para o Estado de São Paulo. Campinas, 1997. p. 119-120. (Boletim Técnico, 100).

REIS JR., R. A.; MONNERAT, P. H. Sugarcane nutritional diagnosis with DRIS norms established in Brazil, South Africa, and the United States. Journal of Plant Nutrition, New York, v. 25, p. 2831-2851, 2002.

RODRÍGUEZ, V.; SILVA, A.; RODRÍGUEZ, O. Balance nutricional y número de hojas como variables de prediccíon del rendimiento del plátano Hartón. Pesquisa Agropecuária Brasileira, Brasília, v. 40, p. 175-177, 2005.

ROZANE, D. E.; PRADO, R. M.; BARBOSA, J. C.; NATALE, W. Tamanho da amostra foliar para avaliação do estado nutricional de goiabeiras com e sem irrigação. Revista Brasileira de Engenharia Agrícola e Ambiental, Campina Grande, v. 13, p. 233-239, 2009.

SANTANA, J. G.; LEANDRO, W. M.; NAVES, R. V.; CUNHA, P. R. Normas DRIS para interpretação de análises de folha e solo, em laranjeira pêra, na região central de Goiás. Pesquisa Agropecuária Tropical, Goiânia, v. 38, p. 109-117, 2008.

SANTOS, A. L.; MONNERAT, P. H.; CARVALHO, A. J. C. Estabelecimento de normas DRIS para o diagnóstico do coqueiro-anão verde na região Norte Fluminense. Revista Brasileira de Fruticultura, Jaboticabal, v. 26, p. 330-334, 2004.

SANTOS, R. R.; QUAGGIO, J. A. Goiaba. In: IAC. Recomendações de adubação e calagem para o Estado de São Paulo. Campinas, 1997. p. 143-144. (Boletim Técnico, 100).

SERRA, A. P.; MARCHETTI, M. E.; ROJAS, E. P.; VITORINO, A. C. T. Beaufils ranges to assess the cotton nutrient status in the southern region Mato Grosso. Revista Brasileira de Ciência do Solo, Viçosa, v. 36, p. 171-181, 2012.
SILVA, G. G. C., NEVES, J. C. L.; ALVAREZ V., V. H. Avaliação da universalidade das normas DRIS, M- DRIS e CND. Revista Brasileira de Ciência do Solo, Viçosa, v. 29, p. 755-761, 2005.

SOUZA, H. A.; NATALE, W.; ROZANE, D. E. Avaliação agronômica da aplicação do resíduo da indústria processadora de goiabas em pomar comercial de goiabeiras. Revista Brasileira de Ciência do Solo, Viçosa, v. 35, p. 969-979, 2011.

SOUZA, H. A.; ROZANE, D. E.; ROMUALDO, L. M.; NATALE, W. Efeitos de diferentes tipos de poda nos teores de nutrientes em flores e frutos de goaibeira. Idesia, Arica, v. 30, p. 45-51, 2012.

TEIXEIRA, L. A. J.; ZAMBROSI, F. C. B.; BETTIOL NETO, J. E. Avaliação do estado nutricional de bananeiras do subgrupo Cavendish no estado de São Paulo: normas DRIS e níveis críticos de nutrientes. Revista Brasileira de Fruticultura, Jaboticabal, v. 29, p. 613-620, 2007.

TERRA, M. M.; GERGOLETTI, I. F.; PIRES, E. J. P.; BOTELHO, R. V.; SANTOS, W. R.; TECCHIO, M. A. Avaliação do estado nutricional da videira 'Itália' na região de São Miguel Arcanjo-SP, utilizando o sistema integrado de diagnose e recomendação. Revista Brasileira de Fruticultura, Jaboticabal, v. 29, p. 710-716, 2007.

URANO, E. M. O.; KURIHARA, C. H.; MAEDA, S.; VITORINO, A. C. T.; GONÇALVES, M. C.; MARCHETTI, M. C. Avaliação do estado nutricional da soja. Pesquisa Agropecuária Brasileira, Brasília, v. 41, p. 1.421-1.428, 2006.

WADT, P. G. S. Relationships between soil class and nutritional status of coffee plantations. Revista Brasileira de Ciência do Solo, v. 29, p. 227-234, 2005.

WADT, P. G. S.; NOVAIS, R. F.; ALVAREZ V., V. H.; FONSECA, S.; BARROS, N. F.; DIAS, L. E. Três métodos de cálculo do DRIS para avaliar o potencial de resposta à adubação de árvores de eucalipto. Revista Brasileira de Ciência do Solo, Viçosa, v. 22, p. 661-666, 1998. 